\title{
Nernst heat theorem for the thermal Casimir interaction between two graphene sheets
}

\author{
V. B. Bezerra, ${ }^{1}$ G. L. Klimchitskaya, ${ }^{2,3,1}$ V. M. Mostepanenko, ${ }^{2,3,4,1}$ and C. Romero ${ }^{1}$ \\ ${ }^{1}$ Department of Physics, Federal University of Paraíba, \\ C.P.5008, CEP 58059-970, João Pessoa, Pb-Brazil \\ ${ }^{2}$ Central Astronomical Observatory at Pulkovo of the Russian \\ Academy of Sciences, Saint Petersburg, 196140, Russia \\ ${ }^{3}$ Institute of Physics, Nanotechnology and Telecommunications, \\ Peter the Great Saint Petersburg Polytechnic University, Saint Petersburg, 195251, Russia \\ ${ }_{4}^{4}$ Kazan Federal University, Kazan, 420008, Russia
}

\begin{abstract}
We find analytic asymptotic expressions at low temperature for the Casimir free energy, entropy and pressure of two parallel graphene sheets in the framework of the Lifshitz theory. The reflection coefficients of electromagnetic waves on graphene are described on the basis of first principles of quantum electrodynamics at nonzero temperature using the polarization tensor in $(2+1)$-dimensional space-time. The leading contributions to the Casimir entropy and to the thermal corrections to the Casimir energy and pressure are given by the thermal correction to the polarization tensor at nonzero Matsubara frequencies. It is shown that the Casimir entropy for two graphene sheets goes to zero when the temperature vanishes, i.e., the third law of thermodynamics (the Nernst heat theorem) is satisfied. At low temperature, the magnitude of the thermal correction to the Casimir pressure between two graphene sheets is shown to vary inversely proportional to the separation. The Nernst heat theorem for graphene is discussed in the context of problems occurring in Casimir physics for both metallic and dielectric plates.
\end{abstract}

PACS numbers: 


\section{INTRODUCTION}

The Casimir interaction arises between two closely spaced material bodies due to fluctuations of the electromagnetic field [1]. At zero temperature, this interaction is caused by the zero-point photons. At nonzero temperature, there is one more contribution to the Casimir interaction due to the thermal photons. Theoretical description of the thermal Casimir force determined by both the zero-point and thermal photons is given by the Lifshitz theory [1, 2]. In the context of this theory, the free energy of the Casimir interaction is presented as a functional of the frequency-dependent dielectric permittivities of material bodies.

During the last few years, the Casimir effect has attracted considerable interest due to the important role it plays in different fields of physics. In atomic physics, it determines the atom-wall interaction and should be taken into account in the phenomena of quantum reflection and Bose-Einstein condensation [3-8]. In condensed matter physics, the Casimir effect was investigated in connection with the role of temperature, conductivity properties, surface roughness and phase transitions [9-13]. There are also prospective applications of the Casimir effect in nanotechnology to actuate micromechanical systems, to explain stiction phenomena, to create new types of microchips, etc. [14-18].

After the advent of graphene a lot of theoretical work has been done to calculate the Casimir free energy and force between two graphene sheets, between a graphene sheet and a plate made of ordinary material, between two graphene-coated substrates, and between an atom and a graphene sheet [19 33]. It was found that, as opposed to ordinary materials, the Casimir force between two graphene sheets has large thermal contribution at short separations of about $100 \mathrm{~nm}$ [20] (for ordinary materials the thermal contribution becomes dominant at separations of about $6 \mu \mathrm{m}[1])$. This is explained by the fact that at low energies the quasiparticles in graphene are massless Dirac fermions, which are described by the linear dispersion relation, but move with the Fermi velocity $v_{F}$ rather than with the speed of light $c$ [34]. The major contribution to the thermal Casimir free energy and force between two graphene sheets is given by the transverse magnetic (TM), i.e., p-polarized, electromagnetic waves. This is akin to the configuration of two metallic plates described by the Drude model. In that case, there is a relatively large thermal effect at short separations, and the contribution of the transverse electric (TE), i.e., s-polarized, electromagnetic waves vanishes for plate separations above $6 \mu \mathrm{m}$. The question arises whether the Casimir free 
energy and entropy of two graphene sheets meet the basic requirements of thermodynamics and, specifically, the Nernst heat theorem.

The point is that there is an outstanding unresolved problem in Lifshitz theory [1, 35]. For metallic bodies made of both nonmagnetic and magnetic metals with perfect crystal lattices, it was shown [36-40] that if the low-frequency behavior of the dielectric permittivity of plate metals is described by the dissipative Drude model, the Casimir free energy and entropy calculated using the Lifshitz theory violate the third law of thermodynamics known as the Nernst heat theorem. In doing so, the Casimir entropy at zero temperature takes a nonzero value which depends on the parameters of the system. If some nonzero relaxation at zero temperature is preserved due to the role of impurities, the Casimir entropy abruptly jumps to zero at very low temperature [41 43], so that the Nernst theorem is formally restored. This, however, does not solve the problem because a perfect crystal lattice is a truly equilibrium system with the nondegenerate state of lowest energy, so that, according to quantum statistical physics, the Nernst heat theorem must be applicable to it [1, 35]. It was shown also that if a lossless plasma model is used instead of the Drude one, the Lifshitz theory is found in perfect agreement with the Nernst heat theorem [36-40].

It was noted [44] that when the spatially nonlocal dielectric response is considered, the Nernst heat theorem is satisfied because the effects of spatial dispersion lead to an effective residual relaxation even for a perfect crystal lattice. At sufficiently short separations, however, the frequency region of infrared optics, where the dielectric response is local, plays a major role. As a result, when the frequency regions with both nonlocal and local response functions are taken into account, the Nernst heat theorem is again violated [45].

As for experiment, all precise measurements of the Casimir interaction between nonmagnetic $(\mathrm{Au})$ and magnetic $(\mathrm{Ni})$ test bodies exclude theoretical predictions of the Lifshitz theory using the Drude model and are in good agreement with those using the plasma model [46 52]. Recently, differential Casimir experiments have been proposed [53 55], where the theoretical predictions calculated with the help of the Drude and plasma models differ by up to a factor of 1000. According to the results of one of these experiments, already performed, the Drude model is unequivocally ruled out by the measurement data, whereas the plasma model shows a good agreement with the data [56, 57]. These results can be considered as surprising because in real electromagnetic fields with a nonzero expectation value the low-frequency response of metals is undoubtedly described by the dissipative Drude model. 
This fact is confirmed by thousands of different experiments. Thus, one can guess that the response of metals to quantum fluctuations, having zero expectation value, might be different.

For dielectric plates, the Lifshitz theory violates the Nernst heat theorem if the dc conductivity of plate materials is taken into account in calculations [58 62]. If the de conductivity is disregarded, the Lifshitz theory is proved to be in perfect agreement with the Nernst heat theorem [58 62]. The experimental data of several precise experiments on measuring the Casimir-Polder and Casimir interaction with dielectric plates [63 66] were found in agreement with theory if the dc conductivity of plate materials is disregarded in calculations. If, however, the dc conductivity of dielectric plates is taken into account, the theoretical results are excluded by the measurement data [64 67]. This is again surprising because at nonzero temperature the conductivity of dielectrics at a constant current is, although small, a really existing and measurable effect. We emphasize, however, that for both dielectrics and metals the experimentally consistent calculations are always found to be in agreement with the Nernst heat theorem. Because of this, the latter can be considered as some kind of test for any novel theoretical approach.

In this paper, we derive the low-temperature asymptotic behavior of the Casimir free energy, entropy and pressure in the configuration of two parallel graphene sheets. As already mentioned, the Casimir effect for two graphene sheets was investigated by many authors and several unusual properties, as compared to metallic plates, have been discovered. In the framework of the Dirac model, the most fundamental description of the response of graphene to electromagnetic field is given by the polarization tensor in $(2+1)$-dimensional space-time. At zero temperature, the polarization tensor of graphene was found in Ref. [19]. At nonzero temperature it was derived in Ref. [24] at the discrete Matsubara frequencies (the comparison with other theoretical approaches to the Casimir effect in graphene systems is contained in Refs. [29, 30]). The Lifshitz theory with the exact reflection coefficients expressed via the polarization tensor [19, 24] has been used in many theoretical papers [27-32, 68, 69]. This formalism was applied in the first experiment on measuring the Casimir interaction between a Au-coated sphere and a graphene-coated substrate [70], and a very good agreement with the measurement results was obtained [71].

The derivation of our asymptotic expressions has been made possible by the use of another representation for the polarization tensor of graphene valid along the entire plane of 
complex frequency [72]. This representation was already exploited to investigate the origin of large thermal effect in graphene systems [33], the reflectivity properties of graphene with a nonzero mass-gap parameter 73] and graphene-coated substrates [74], and the conductivity properties of graphene [75]. According to our results, with vanishing temperature $T$ the leading contributions to thermal correction to the Casimir energy and to the Casimir entropy behave as $\sim T^{3} \ln T$ and $\sim T^{2} \ln T$, respectively. This means that the Lifshitz theory for graphene is in agreement with the Nernst heat theorem.

The paper is organized as follows. In Sec. II, we present the general formalism of the Lifshitz theory using the polarization tensor of graphene at low temperature. In Sec. III, the thermal correction to the Casimir energy is considered, which arises due to a summation over the discrete Matsubara frequencies when the zero-temperature polarization tensor is used in calculations. Section IV is devoted to the thermal correction arising due to the temperature dependence of the polarization tensor. We consider separately contributions from the zerofrequency term and from all terms with nonzero Matsubara frequencies. The total results for the low-temperature behavior of the Casimir free energy, entropy and pressure for two graphene sheets are presented in Sec. V. Section VI contains our conclusions and discussion. Some details of mathematical derivations are given in Appendix A.

\section{GENERAL FORMALISM IN TERMS OF THE POLARIZATION TENSOR OF GRAPHENE AT LOW TEMPERATURE}

We consider the Casimir free energy per unit area of two parallel graphene sheets separated by a gap of thickness $a$ at temperature $T$ in thermal equilibrium with the environment $[1,[2]$

$$
\mathcal{F}(a, T)=\frac{k_{B} T}{2 \pi} \sum_{l=0}^{\infty} \int_{0}^{\infty} k_{\perp} d k_{\perp}\left[\ln \left(1-r_{\mathrm{TM}}^{2} e^{-2 q_{l} a}\right)+\ln \left(1-r_{\mathrm{TE}}^{2} e^{-2 q_{l} a}\right)\right] .
$$

Here, $k_{B}$ is the Boltzmann constant, the prime on the summation sign means that the term with $l=0$ is divided by $2, k_{\perp}$ is the magnitude of the projection of the wave vector on the plane of plates, and

$$
q_{l}=\sqrt{k_{\perp}^{2}+\frac{\xi_{l}^{2}}{c^{2}}}
$$

where $\xi_{l}=2 \pi k_{B} T l / \hbar$ are the Matsubara frequencies. 
The reflection coefficients $r_{\mathrm{TM}, \mathrm{TE}}$ can be expressed via the components of the polarization tensor of graphene, $\Pi_{m n}$, with $m, n=0,1,2$ [19, 24, 72]

$$
\begin{aligned}
& r_{\mathrm{TM}} \equiv r_{\mathrm{TM}}\left(i \xi_{l}, k_{\perp}\right)=\frac{q_{l} \Pi_{00}\left(i \xi_{l}, k_{\perp}\right)}{q_{l} \Pi_{00}\left(i \xi_{l}, k_{\perp}\right)+2 \hbar k_{\perp}^{2}}, \\
& r_{\mathrm{TE}} \equiv r_{\mathrm{TE}}\left(i \xi_{l}, k_{\perp}\right)=-\frac{\Pi\left(i \xi_{l}, k_{\perp}\right)}{\Pi\left(i \xi_{l}, k_{\perp}\right)+2 \hbar k_{\perp}^{2} q_{l}},
\end{aligned}
$$

where the combination of the components $\Pi$ is defined as

$$
\Pi\left(i \xi_{l}, k_{\perp}\right)=k_{\perp}^{2} \Pi_{\mathrm{tr}}\left(i \xi_{l}, k_{\perp}\right)-q_{l}^{2} \Pi_{00}\left(i \xi_{l}, k_{\perp}\right)
$$

and $\Pi_{\mathrm{tr}} \equiv \Pi_{m}^{m}$.

Note that the polarization tensor is directly connected with the longitudinal and transverse nonlocal dielectric permittivities of graphene [25, $\underline{30}, 76]$

$$
\begin{aligned}
& \varepsilon_{\|}\left(i \xi_{l}, k_{\perp}\right)=1+\frac{1}{2 \hbar k_{\perp}} \Pi_{00}\left(i \xi_{l}, k_{\perp}\right), \\
& \varepsilon_{\perp}\left(i \xi_{l}, k_{\perp}\right)=1+\frac{c^{2}}{2 \hbar k_{\perp} \xi_{l}^{2}} \Pi\left(i \xi_{l}, k_{\perp}\right) .
\end{aligned}
$$

Thus, for graphene the dielectric permittivity is calculated precisely starting from first principles of quantum field theory at nonzero temperature. This is different from more complicated materials which are usually described by phenomenological dielectric functions.

For obtaining the low-temperature asymptotic expressions for the Casimir free energy, it is convenient to use the representation of the polarization tensor obtained in Ref. [72] and applied in Refs. [33, 73-75] (see also Ref. [77], where this representation was generalized for the case of nonzero chemical potential). At first, the quantities $\Pi_{00}$ and $\Pi$ are presented as the sums of zero-temperature contributions and the thermal corrections to them

$$
\begin{aligned}
& \Pi_{00}\left(i \xi, k_{\perp}\right)=\Pi_{00}^{(0)}\left(i \xi, k_{\perp}\right)+\Delta_{T} \Pi_{00}\left(i \xi, k_{\perp}\right), \\
& \Pi\left(i \xi, k_{\perp}\right)=\Pi^{(0)}\left(i \xi, k_{\perp}\right)+\Delta_{T} \Pi\left(i \xi, k_{\perp}\right) .
\end{aligned}
$$

Note that for arbitrary $\xi$ the quantities $\Pi_{00}^{(0)}$ and $\Pi^{(0)}$ are temperature independent. If, however, $\xi$ is replaced with $\xi_{l}$, they depend on $T$ through the Matsubara frequencies.

Below we consider pure (gapless) graphene sheets. For the zero-temperature contributions in Eq. (6), calculated at the Matsubara frequencies, one has [19, 24, 72$]$

$$
\begin{aligned}
& \Pi_{00}^{(0)}\left(i \xi_{l}, k_{\perp}\right)=\frac{\pi \alpha \hbar k_{\perp}^{2}}{\tilde{q}_{l}}, \\
& \Pi^{(0)}\left(i \xi_{l}, k_{\perp}\right)=\pi \alpha \hbar k_{\perp}^{2} \tilde{q}_{l},
\end{aligned}
$$


where $\alpha=e^{2} /(\hbar c)$ is the fine structure constant,

$$
\tilde{q}_{l}=\sqrt{\tilde{v}_{F}^{2} k_{\perp}^{2}+\frac{\xi_{l}^{2}}{c^{2}}},
$$

and $\tilde{v}_{F}=v_{F} / c \approx 1 / 300$.

The respective zero-temperature reflection coefficients are obtained by the substitution of Eq. (17) in Eq. (3). They have the following form:

$$
\begin{aligned}
& r_{\mathrm{TM}}^{(0)} \equiv r_{\mathrm{TM}}^{(0)}\left(i \xi_{l}, k_{\perp}\right)=\frac{q_{l} \Pi_{00}^{(0)}\left(i \xi_{l}, k_{\perp}\right)}{q_{l} \Pi_{00}^{(0)}\left(i \xi_{l}, k_{\perp}\right)+2 \hbar k_{\perp}^{2}}=\frac{\alpha \pi q_{l}}{\alpha \pi q_{l}+2 \tilde{q}_{l}}, \\
& r_{\mathrm{TE}}^{(0)} \equiv r_{\mathrm{TE}}^{(0)}\left(i \xi_{l}, k_{\perp}\right)=-\frac{\Pi^{(0)}\left(i \xi_{l}, k_{\perp}\right)}{\Pi^{(0)}\left(i \xi_{l}, k_{\perp}\right)+2 \hbar k_{\perp}^{2} q_{l}}=-\frac{\alpha \pi \tilde{q}_{l}}{\alpha \pi \tilde{q}_{l}+2 q_{l}} .
\end{aligned}
$$

The thermal corrections on the right-hand side of Eq. (66) at the Matsubara frequencies are most conveniently represented in Eq. (10) of Ref. [33]

$$
\begin{aligned}
& \Delta_{T} \Pi_{00}\left(i \xi_{l}, k_{\perp}\right)=\frac{8 \alpha \hbar \tilde{q}_{l}}{\tilde{v}_{F}^{2}} \int_{0}^{\infty} \frac{d u}{e^{B_{l} u}+1} \\
& \quad \times\left\{1-\frac{1}{\sqrt{2}}\left[\sqrt{\left(1+u^{2}\right)^{2}-4 \frac{\tilde{v}_{F}^{2} k_{\perp}^{2} u^{2}}{\tilde{q}_{l}^{2}}}+1-u^{2}\right]^{1 / 2}\right\}, \\
& \Delta_{T} \Pi\left(i \xi_{l}, k_{\perp}\right)=\frac{8 \alpha \hbar \tilde{q}_{l}}{\tilde{v}_{F}^{2}} \int_{0}^{\infty} \frac{d u}{e^{B_{l} u}+1} \\
& \times\left\{-\frac{\xi_{l}^{2}}{c^{2}}+\frac{\tilde{q}_{l}^{2}}{\sqrt{2}}\left[\sqrt{\left(1+u^{2}\right)^{2}-4 \frac{\tilde{v}_{F}^{2} k_{\perp}^{2} u^{2}}{\tilde{q}_{l}^{2}}}+1-u^{2}\right]^{1 / 2}\right. \\
& \left.\times\left[1-\frac{\tilde{v}_{F}^{2} k_{\perp}^{2}}{\tilde{q}_{l}^{2} \sqrt{\left(1+u^{2}\right)^{2}-4 \frac{\tilde{v}_{F}^{2} k_{\perp}^{2} u^{2}}{\tilde{q}_{l}^{2}}}}\right]\right\}
\end{aligned}
$$

where $B_{l} \equiv \hbar c \tilde{q}_{l} /\left(2 k_{B} T\right)$.

As is seen from Eq. (10),

$$
\lim _{T \rightarrow 0} \Delta_{T} \Pi_{00}\left(i \xi_{l}, k_{\perp}\right)=\lim _{T \rightarrow 0} \Delta_{T} \Pi\left(i \xi_{l}, k_{\perp}\right)=0
$$

whereas for the zero-temperature contributions calculated at the Matsubara frequencies one has:

$$
\begin{aligned}
& \lim _{T \rightarrow 0} \Pi_{00}^{(0)}\left(i \xi_{l}, k_{\perp}\right)=\frac{\pi \alpha \hbar k_{\perp}}{\tilde{v}_{F}} \neq 0, \\
& \lim _{T \rightarrow 0} \Pi^{(0)}\left(i \xi_{l}, k_{\perp}\right)=\pi \alpha \hbar \tilde{v}_{F} k_{\perp}^{3} \neq 0 .
\end{aligned}
$$


Thus, for sufficiently low $T$, one can use two small parameters, namely,

$$
\frac{\Delta_{T} \Pi_{00}}{\Pi_{00}^{(0)}} \ll 1, \quad \frac{\Delta_{T} \Pi}{\Pi^{(0)}} \ll 1 .
$$

An explicit condition on the smallness of $T$, wherein the inequalities (13) are satisfied, is determined in Secs. III and IV.

Now, we substitute Eq. (6) in Eq. (3), expand in powers of the small parameters (13) and preserve only the first-order terms. Then, using Eq. (9), we obtain

$$
\begin{aligned}
& r_{\mathrm{TM}}=r_{\mathrm{TM}}^{(0)}+\frac{2 \hbar k_{\perp}^{2} r_{\mathrm{TM}}^{(0)}}{q_{l} \Pi_{00}^{(0)}+2 \hbar k_{\perp}^{2}} \frac{\Delta_{T} \Pi_{00}}{\Pi_{00}^{(0)}}, \\
& r_{\mathrm{TE}}=r_{\mathrm{TE}}^{(0)}+\frac{2 \hbar k_{\perp}^{2} q_{l} r_{\mathrm{TE}}^{(0)}}{\Pi^{(0)}+2 \hbar k_{\perp}^{2} q_{l}} \frac{\Delta_{T} \Pi}{\Pi^{(0)}} .
\end{aligned}
$$

Taking the square of Eq. (14) and using Eq. (7), in the first order of the small parameters (13) we find

$$
\begin{aligned}
& r_{\mathrm{TM}}^{2}=r_{\mathrm{TM}}^{(0)^{2}}\left(1+\frac{4 \tilde{q}_{l}}{\pi \alpha q_{l}+2 \tilde{q}_{l}} \frac{\Delta_{T} \Pi_{00}}{\Pi_{00}^{(0)}}\right), \\
& r_{\mathrm{TE}}^{2}=r_{\mathrm{TE}}^{(0)^{2}}\left(1+\frac{4 q_{l}}{\pi \alpha \tilde{q}_{l}+2 q_{l}} \frac{\Delta_{T} \Pi}{\Pi^{(0)}}\right) .
\end{aligned}
$$

Substituting Eq. (15) in Eq. (1) and expanding the logarithms up to the first powers of small parameters (13), one arrives at

$$
\begin{aligned}
& \mathcal{F}(a, T) \equiv \mathcal{F}^{(1)}(a, T)+\Delta_{T}^{(2)} \mathcal{F}(a, T) \\
& =\frac{k_{B} T}{2 \pi} \sum_{l=0}^{\infty} \int_{0}^{\infty} k_{\perp} d k_{\perp}\left[\ln \left(1-r_{\mathrm{TM}}^{(0)^{2}} e^{-2 q_{l} a}\right)+\ln \left(1-r_{\mathrm{TE}}^{(0)^{2}} e^{-2 q_{l} a}\right)\right] \\
& -\frac{2 k_{B} T}{\pi} \sum_{l=0}^{\infty} \int_{0}^{\infty} k_{\perp} d k_{\perp}\left[\frac{\tilde{q}_{l}}{\pi \alpha q_{l}+2 \tilde{q}_{l}} \frac{r_{\mathrm{TM}}^{(0)^{2}}}{e^{2 q_{l} a}-r_{\mathrm{TM}}^{(0)^{2}}} \frac{\Delta_{T} \Pi_{00}}{\Pi_{00}^{(0)}}\right. \\
& \left.\quad+\frac{q_{l}}{\pi \alpha \tilde{q}_{l}+2 q_{l}} \frac{\left.r_{\mathrm{TE}}^{(0)}\right)^{2 q_{l} a}-r_{\mathrm{TE}}^{(0)^{2}}}{e_{T} \Pi} \frac{\Delta_{T} \Pi}{\Pi^{(0)}}\right]
\end{aligned}
$$

where $\mathcal{F}^{(1)}$ and $\Delta_{T}^{(2)} \mathcal{F}$ are equal to the first and second primed sums, respectively.

Now it is essential to have in mind the Lifshitz formula for the Casimir energy per unit area of graphene sheet at zero temperature, which reads as [1]

$$
E(a)=\frac{\hbar}{4 \pi^{2}} \int_{0}^{\infty} d \xi \int_{0}^{\infty} k_{\perp} d k_{\perp}\left[\ln \left(1-r_{\mathrm{TM}}^{(0)^{2}} e^{-2 q a}\right)+\ln \left(1-r_{\mathrm{TE}}^{(0)^{2}} e^{-2 q a}\right)\right]
$$


where

$$
r_{\mathrm{TM}, \mathrm{TE}}^{(0)} \equiv r_{\mathrm{TM}, \mathrm{TE}}^{(0)}\left(i \xi, k_{\perp}\right), \quad q=\sqrt{k_{\perp}^{2}+\frac{\xi^{2}}{c^{2}}}
$$

are functions of the continuous variable $\xi$.

It is seen that the first sum in Eq. (16) is obtained from Eq. (17) by the familiar substitution

$$
\frac{\hbar}{4 \pi^{2}} \int_{0}^{\infty} d \xi \rightarrow k_{B} T \sum_{l=0}^{\infty}
$$

Because of this, it is convenient to represent this sum in the form

$$
\mathcal{F}^{(1)}(a, T)=E(a)+\Delta_{T}^{(1)} \mathcal{F}(a, T),
$$

where $\Delta_{T}^{(1)} \mathcal{F}(a, T)$ has the meaning of the first part of the thermal correction to the Casimir energy of two graphene sheets. The thermal correction $\Delta_{T}^{(1)} \mathcal{F}(a, T)$ is determined by the fact that at nonzero $T$ the continuous argument $\xi$ in the zero-temperature polarization tensor $\Pi_{m n}^{(0)}$ and the respective reflection coefficients $r_{\mathrm{TM}, \mathrm{TE}}^{(0)}$ is replaced by the discrete Matsubara frequencies $\xi_{l}$, and originates from a summation over these frequencies.

The second sum in Eq. (16), which we have notated $\Delta_{T}^{(2)} \mathcal{F}(a, T)$, has the meaning of the second part of thermal correction to the Casimir energy of two graphene sheets. It originates from an explicit dependence of the polarization tensor $\Pi_{m n}$ on the temperature as a parameter. With account of Eq. (20), Eq. (16) can be rewritten as

$$
\mathcal{F}(a, T)=E(a)+\Delta_{T}^{(1)} \mathcal{F}(a, T)+\Delta_{T}^{(2)} \mathcal{F}(a, T) .
$$

Below it is convenient to determine the individual asymptotic behaviors of each part of the total thermal correction

$$
\Delta_{T} \mathcal{F}(a, T)=\Delta_{T}^{(1)} \mathcal{F}(a, T)+\Delta_{T}^{(2)} \mathcal{F}(a, T)
$$

at low temperature separately. This is done in the next two sections.

\section{THERMAL CORRECTION TO THE CASIMIR ENERGY DUE TO SUMMATION OVER THE MATSUBARA FREQUENCIES}

In this section, we investigate the low-temperature behavior of the thermal correction $\Delta_{T}^{(1)} \mathcal{F}$ defined in Eq. (20). It is convenient to represent the quantity $\mathcal{F}^{(1)}(a, T)$ in terms of 
the dimensionless variables

$$
y=2 a q_{l}, \quad \zeta_{l}=\frac{\xi_{l}}{\omega_{c}} \equiv \frac{2 a \xi_{l}}{c} \equiv \tau l
$$

where

$$
\tau=4 \pi \frac{a k_{B} T}{\hbar c}=2 \pi \frac{T}{T_{\mathrm{eff}}}, \quad k_{B} T_{\mathrm{eff}} \equiv \hbar \omega_{c} .
$$

Then, using Eq. (16), one arrives at

$$
\mathcal{F}^{(1)}=\frac{k_{B} T}{8 \pi a^{2}} \sum_{l=0}^{\infty}{ }^{\prime} \Phi(\tau l)
$$

where

$$
\Phi(\tau l)=\int_{\tau l}^{\infty} y d y\left[\ln \left(1-r_{\mathrm{TM}}^{(0)^{2}} e^{-y}\right)+\ln \left(1-r_{\mathrm{TE}}^{(0)^{2}} e^{-y}\right)\right]
$$

and the reflection coefficients (9) take the form

$$
r_{\mathrm{TM}}^{(0)}=\frac{\alpha \pi y}{\alpha \pi y+2 \tilde{g}_{l}}, \quad r_{\mathrm{TE}}^{(0)}=\frac{\alpha \pi \tilde{g}_{l}}{\alpha \pi \tilde{g}_{l}+2 y} .
$$

Here, the dimensionless function $\tilde{g}_{l}$ is defined as

$$
\tilde{g}_{l}=2 a \tilde{q}_{l}=\sqrt{\tilde{v}_{F}^{2} y^{2}+\left(1-\tilde{v}_{F}^{2}\right)(\tau l)^{2}} \approx \sqrt{\tilde{v}_{F}^{2} y^{2}+(\tau l)^{2}},
$$

where we have neglected the small quantity $\tilde{v}_{F}^{2}$ as compared to unity. Now we represent the Casimir energy at zero temperature, $E(a)$, in terms of dimensionless variables

$$
y=2 a q, \quad \zeta=\frac{\xi}{\omega_{c}} \equiv \tau l
$$

where $q$ is defined in Eq. (18) and $\tau$ in Eq. (24). Then, from Eq. (17) one obtains

$$
E(a)=\frac{k_{B} T}{8 \pi a^{2}} \int_{0}^{\infty} d t \Phi(\tau t) .
$$

Here, the function $\Phi$ is defined in Eqs. (26) and (27), where the discrete quantity $l$ is replaced with the continuous variable $t$.

From the comparison of Eqs. (25) and (30) it is seen that the Casimir energy has the same form as the quantity $\mathcal{F}^{(1)}$, but is represented by an integral instead of a discrete sum. Then, the thermal correction $\Delta_{T}^{(1)} \mathcal{F}(a, T)$, defined in Eq. (20), can be found as the difference between the sum and the integral

$$
\Delta_{T}^{(1)} \mathcal{F}(a, T)=\frac{k_{B} T}{8 \pi a^{2}}\left[\sum_{l=0}^{\infty}{ }^{\prime} \Phi(\tau l)-\int_{0}^{\infty} d t \Phi(\tau t)\right] .
$$


Using the Abel-Plana formula [1], we can rewrite Eq. (31) in the form

$$
\Delta_{T}^{(1)} \mathcal{F}(a, T)=\frac{i k_{B} T}{8 \pi a^{2}} \int_{0}^{\infty} d t \frac{\Phi(i \tau t)-\Phi(-i \tau t)}{e^{2 \pi t}-1} .
$$

The major contribution to this integral is given by $t \sim 1 /(2 \pi)$. At the same time, the major contribution to the integral (26) is given by $y \sim 1$. Below, we consider sufficiently low $T$ such that for $t \sim 1 /(2 \pi)$ and $y \sim 1$ one would have $\tau t \ll \tilde{v}_{F} y$, i.e., in accordance with Eq. (24),

$$
\frac{\tau}{2 \pi}=\frac{T}{T_{\text {eff }}} \ll \tilde{v}_{F}
$$

This inequality can be rewritten in the form

$$
k_{B} T \ll \frac{\hbar v_{F}}{2 a} \equiv k_{B} T_{\mathrm{eff}}^{(g)}
$$

Here, $T_{\mathrm{eff}}^{(g)}$ is the effective temperature for graphene, where, as compared to the standard definition (24), the speed of light $c$ is replaced with the Fermi velocity $v_{F}[20]$. Note that the region of $T$ defined in Eqs. (33) and (34) depends on the separation distance between two graphene sheets. For example, for $a=10$ and $100 \mathrm{~nm}$ Eqs. (33) and (34) lead to $T \ll 300 \mathrm{~K}$ and $T \ll 30 \mathrm{~K}$, respectively.

Expanding the logarithms in the definition of $\Phi$ in a power series, one obtains

$$
\begin{aligned}
& \Phi(\tau t) \equiv \Phi_{\mathrm{TM}}(\tau t)+\Phi_{\mathrm{TE}}(\tau t) \\
& =-\sum_{n=1}^{\infty} \frac{1}{n} \int_{\tau t}^{\infty} y d y\left[r_{\mathrm{TM}}^{(0)}{ }^{2 n}(i \tau t, y)+r_{\mathrm{TE}}^{(0)}{ }^{2 n}(i \tau t, y)\right] e^{-n y}
\end{aligned}
$$

where $\Phi_{\mathrm{TM}}$ and $\Phi_{\mathrm{TE}}$ are defined via the respective reflection coeffecients $r_{\mathrm{TM}}^{(0)}$ and $r_{\mathrm{TE}}^{(0)}$.

Now we expand the powers of the reflection coefficients (27), appearing in Eq. (35), in powers of the small parameter $\tau t /\left(\tilde{v}_{F} y\right)$ by preserving only the lowest order contribution

$$
\begin{aligned}
& \left.r_{\mathrm{TM}}^{(0)}\right)^{2 n}(i \tau t, y)=\rho_{\mathrm{TM}}^{2 n}\left[1-2 n \frac{\tilde{v}_{F}}{\alpha \pi+2 \tilde{v}_{F}}\left(\frac{\tau t}{\tilde{v}_{F} y}\right)^{2}\right] \\
& r_{\mathrm{TE}}^{(0)^{2 n}}(i \tau t, y)=\rho_{\mathrm{TE}}^{2 n}\left[1+2 n \frac{1}{2+\alpha \pi \tilde{v}_{F}}\left(\frac{\tau t}{\tilde{v}_{F} y}\right)^{2}\right]
\end{aligned}
$$

The quantities $\rho_{\mathrm{TM}, \mathrm{TE}}$, introduced here, are defined as

$$
\begin{aligned}
& \rho_{\mathrm{TM}}=\frac{\alpha \pi}{\alpha \pi+2 \tilde{v}_{F}}, \quad \rho_{\mathrm{TM}}^{2} \approx 0.6 \\
& \rho_{\mathrm{TE}}=\frac{\alpha \pi \tilde{v}_{F}}{2+\alpha \pi \tilde{v}_{F}} \approx \frac{\alpha \pi \tilde{v}_{F}}{2}, \quad \rho_{\mathrm{TE}}^{2} \approx 1.4 \times 10^{-9} .
\end{aligned}
$$


Note that these coefficients coincide with those in Eq. (27) for $l=0$.

Substituting Eqs. (36) and (37) in Eq. (35), we arrive at

$$
\begin{aligned}
& \Phi_{\mathrm{TM}}(\tau t)=-\sum_{n=1}^{\infty} \frac{\rho_{\mathrm{TM}}^{2 n}}{n}\left[R_{n}^{(1)}(\tau t)-\frac{2 n(\tau t)^{2}}{\tilde{v}_{F}\left(\alpha \pi+2 \tilde{v}_{F}\right)} R_{n}^{(2)}(\tau t)\right], \\
& \Phi_{\mathrm{TE}}(\tau t)=-\rho_{\mathrm{TE}}^{2}\left[R_{1}^{(1)}(\tau t)+\frac{(\tau t)^{2}}{\tilde{v}_{F}^{2}} R_{1}^{(2)}(\tau t)\right],
\end{aligned}
$$

where the functions $R_{n}^{(1)}$ and $R_{n}^{(2)}$ are defined as

$$
\begin{aligned}
& R_{n}^{(1)}(\tau t) \equiv \int_{\tau t}^{\infty} y d y e^{-n y}=\frac{1}{n^{2}} e^{-n \tau t}(1+n \tau t), \\
& R_{n}^{(2)}(\tau t) \equiv \int_{\tau t}^{\infty} \frac{d y}{y} e^{-n y}=-\operatorname{Ei}(-n \tau t),
\end{aligned}
$$

and $\operatorname{Ei}(z)$ is the integral exponent function [78]. Note that in the second line of Eq. (38) we keep only the first term in the sum with respect to $n$. This is justified by the smallness of the quantity $\rho_{\mathrm{TE}}^{2}$, in accordance to Eq. (37).

Expanding the right-hand sides in Eq. (39) in powers of $n \tau t$ one obtains

$$
\begin{aligned}
& R_{n}^{(1)}(\tau t)=\frac{1}{n^{2}}\left[1-\frac{n^{2}}{2}(\tau t)^{2}+\frac{n^{3}}{3}(\tau t)^{3}+\ldots\right], \\
& R_{n}^{(2)}(\tau t)=-[C+\ln (n \tau t)-n \tau t+\ldots],
\end{aligned}
$$

where $C$ is the Euler constant.

Now we are in the position to calculate the leading term in the difference $\Phi(i \tau t)-\Phi(-i \tau t)$ appearing in Eq. (32). This is done separately for $\Phi_{\mathrm{TM}}$ and $\Phi_{\mathrm{TE}}$. At first, we substitute Eq. (401) in the first line of Eq. (38) and to the leading order in $\tau t$ we find

$$
\begin{gathered}
\Phi_{\mathrm{TM}}(i \tau t)-\Phi_{\mathrm{TM}}(-i \tau t)=\frac{2 \tau^{2} t^{2}}{\tilde{v}_{F}\left(\alpha \pi+2 \tilde{v}_{F}\right)} \sum_{n=1}^{\infty} \rho_{\mathrm{TM}}^{2 n}[\ln i-\ln (-i)] \\
=i \frac{2 \pi \tau^{2} t^{2}}{\tilde{v}_{F}\left(\alpha \pi+2 \tilde{v}_{F}\right)} \frac{\rho_{\mathrm{TM}}^{2}}{1-\rho_{\mathrm{TM}}^{2}} .
\end{gathered}
$$

Note that $R_{n}^{(1)}$ contributes to Eq. (41) only starting from the third-order term in Eq. (40), i.e., would lead to a higher-order correction $\sim \tau^{3}$. In a similar way, the next after the logarithm, linear, term in $R_{n}^{(2)}$ would also lead to a correction of order $\tau^{3}$ and, thus, both these corrections can be omitted.

Likewise, substituting Eq. (40) in the second line of Eq. (38) and omitting the terms of order of $\tau^{3}$ and higher, one finds

$$
\Phi_{\mathrm{TE}}(i \tau t)-\Phi_{\mathrm{TE}}(-i \tau t)=-i \frac{\pi \tau^{2} t^{2}}{\tilde{v}_{F}^{2}} \rho_{\mathrm{TE}}^{2} .
$$


Using Eq. (37), Eqs. (41) and (42) can be rewritten in the form

$$
\begin{aligned}
& \Phi_{\mathrm{TM}}(i \tau t)-\Phi_{\mathrm{TM}}(-i \tau t)=i \frac{\pi^{3} \alpha^{2} \tau^{2} t^{2}}{2 \tilde{v}_{F}^{2}\left(\alpha \pi+2 \tilde{v}_{F}\right)\left(\alpha \pi+\tilde{v}_{F}\right)} \\
& \Phi_{\mathrm{TE}}(i \tau t)-\Phi_{\mathrm{TE}}(-i \tau t)=-i \frac{1}{4} \pi^{3} \alpha^{2} \tau^{2} t^{2} .
\end{aligned}
$$

Substituting these results in Eq. (32), for the TM and TE contributions to the thermal correction $\Delta_{T}^{(1)} \mathcal{F}$, we obtain

$$
\begin{aligned}
& \Delta_{T}^{(1)} \mathcal{F}_{\mathrm{TM}}(a, T)=-\frac{k_{B} T}{16 a^{2}} \frac{\pi^{2} \alpha^{2} \tau^{2}}{\tilde{v}_{F}^{2}\left(\alpha \pi+2 \tilde{v}_{F}\right)\left(\alpha \pi+\tilde{v}_{F}\right)} \int_{0}^{\infty} d t \frac{t^{2}}{e^{2 \pi t}-1}, \\
& \Delta_{T}^{(1)} \mathcal{F}_{\mathrm{TE}}(a, T)=\frac{k_{B} T}{32 a^{2}} \pi^{2} \alpha^{2} \tau^{2} \int_{0}^{\infty} d t \frac{t^{2}}{e^{2 \pi t}-1} .
\end{aligned}
$$

Calculating the integrals in this equation and returning to the dimensional temperature $T$ in all factors, one arrives at

$$
\begin{aligned}
& \Delta_{T}^{(1)} \mathcal{F}_{\mathrm{TM}}(a, T)=-\frac{\left(k_{B} T\right)^{3}}{\left(\hbar v_{F}\right)^{2}} \frac{\zeta(3)}{4} \frac{\pi \alpha^{2}}{\left(\alpha \pi+2 \tilde{v}_{F}\right)\left(\alpha \pi+\tilde{v}_{F}\right)}, \\
& \Delta_{T}^{(1)} \mathcal{F}_{\mathrm{TE}}(a, T)=\frac{\left(k_{B} T\right)^{3}}{(\hbar c)^{2}} \frac{\zeta(3)}{8} \pi \alpha^{2},
\end{aligned}
$$

where $\zeta(z)$ is the Riemann zeta function.

As is seen in Eq. (45), the contributions of the TM and TE modes to the thermal correction $\Delta_{T}^{(1)} \mathcal{F}$ are of the same order in temperature, but of opposite signs. It is seen also that the TE contribution is negligibly small, as compared to the magnitude of the TM contribution

$$
\frac{\Delta_{T}^{(1)} \mathcal{F}_{\mathrm{TE}}}{\left|\Delta_{T}^{(1)} \mathcal{F}_{\mathrm{TM}}\right|}=\frac{1}{2} \tilde{v}_{F}^{2}\left(\alpha \pi+2 \tilde{v}_{F}\right)\left(\alpha \pi+\tilde{v}_{F}\right) \ll 1 .
$$

According to Eq. (45), both contributions to the thermal correction $\Delta_{T}^{(1)} \mathcal{F}$ do not depend on the separation and, thus, do not contribute to the Casimir pressure between two graphene sheets (see Sec. V for further discussion).

At the end of this section, we underline that the higher-order terms in the small parameter $\tau t /\left(\tilde{v}_{F} y\right)$ in Eq. (36), neglected in our calculation, result in corrections of order $\tau^{3} t^{3}$ and higher in the difference $\Phi(i \tau t)-\Phi(-i \tau t)$. These corrections lead to terms of order $T^{4}$, which can be neglected in comparison with the leading terms found in Eq. (45). Note that several applications of the Abel-Plana formula for determination of the low-temperature behavior of the Casimir force between different materials are considered in Ref. [79]. 


\section{THERMAL CORRECTION TO THE CASIMIR ENERGY DUE TO TEMPERATURE DEPENDENCE OF THE POLARIZATION TENSOR}

In this section, we investigate the asymptotic behavior at low $T$ of the second part of the thermal correction to the Casimir energy between two graphene sheets, $\Delta_{T}^{(2)} \mathcal{F}(a, T)$, defined by the second sum in $l$ in Eq. (16). It is convenient to consider separately contributions to $\Delta_{T}^{(2)} \mathcal{F}$ of the zero-frequency term and of all terms with nonzero Matsubara frequencies.

\section{A. Contribution of the zero-frequency term}

According to Eq. (16), the zero-frequency contribution to the second part of the thermal correction $\Delta_{T}^{(2)} \mathcal{F}$ is represented by the sum of TM and TE modes

$$
\Delta_{T}^{(2)} \mathcal{F}^{(l=0)}(a, T)=\Delta_{T}^{(2)} \mathcal{F}_{\mathrm{TM}}^{(l=0)}(a, T)+\Delta_{T}^{(2)} \mathcal{F}_{\mathrm{TE}}^{(l=0)}(a, T)
$$

where

$$
\begin{aligned}
& \Delta_{T}^{(2)} \mathcal{F}_{\mathrm{TM}}^{(l=0)}(a, T)=-\frac{k_{B} T}{\pi} \frac{\tilde{v}_{F}}{\pi \alpha+2 \tilde{v}_{F}} \int_{0}^{\infty} k_{\perp} d k_{\perp} \frac{r_{\mathrm{TM}}^{(0)^{2}}}{e^{2 k_{\perp} a}-r_{\mathrm{TM}}^{(0)}{ }^{2}} \frac{\Delta_{T} \Pi_{00}\left(0, k_{\perp}\right)}{\Pi_{00}^{(0)}\left(0, k_{\perp}\right)} \\
& \Delta_{T}^{(2)} \mathcal{F}_{\mathrm{TE}}^{(l=0)}(a, T)=-\frac{k_{B} T}{\pi} \frac{1}{\pi \alpha \tilde{v}_{F}+2} \int_{0}^{\infty} k_{\perp} d k_{\perp} \frac{r_{\mathrm{TE}}^{(0)^{2}}}{e^{2 k_{\perp} a}-r_{\mathrm{TE}}^{(0)}{ }^{2}} \frac{\Delta_{T} \Pi\left(0, k_{\perp}\right)}{\Pi^{(0)}\left(0, k_{\perp}\right)}
\end{aligned}
$$

The thermal corrections $\Delta_{T} \Pi_{00}\left(0, k_{\perp}\right)$ and $\Delta_{T} \Pi\left(0, k_{\perp}\right)$ are obtained from Eq. (10) by putting $l=0$

$$
\begin{aligned}
& \Delta_{T} \Pi_{00}\left(0, k_{\perp}\right)=\frac{8 \alpha \hbar k_{\perp}}{\tilde{v}_{F}}\left(\int_{0}^{\infty} \frac{d u}{e^{B_{0} u}+1}-\int_{0}^{1} \frac{d u}{e^{B_{0} u}+1} \sqrt{1-u^{2}}\right), \\
& \Delta_{T} \Pi\left(0, k_{\perp}\right)=-8 \alpha \hbar \tilde{v}_{F} k_{\perp}^{3} \int_{0}^{1} \frac{d u}{e^{B_{0} u}+1} \frac{u^{2}}{\sqrt{1-u^{2}}}
\end{aligned}
$$

where $B_{0}=\hbar c \tilde{v}_{F} k_{\perp} /\left(2 k_{B} T\right)$. These expressions coincide up to a change of the integration variable with Eq. (51) of Ref. [72]. As shown in Ref. [72], by means of identical transformations the expressions (49) can be put in an equivalent form

$$
\begin{aligned}
& \Delta_{T} \Pi_{00}\left(0, k_{\perp}\right)=\frac{16 \alpha k_{B} T}{\tilde{v}_{F}^{2} c} \int_{0}^{1} d x \ln \left[1+e^{-2 B_{0} \sqrt{x(1-x)}}\right] \\
& \Delta_{T} \Pi\left(0, k_{\perp}\right)=-16 \alpha \hbar \tilde{v}_{F} k_{\perp}^{3} \int_{0}^{1} \frac{\sqrt{x(1-x)} d x}{e^{2 B_{0} \sqrt{x(1-x)}}+1}
\end{aligned}
$$

which is convenient for us now. 
Substituting Eq. (50) in Eq. (48) and using Eq. (17) at $\xi_{0}=0$, we find

$$
\begin{aligned}
& \Delta_{T}^{(2)} \mathcal{F}_{\mathrm{TM}}^{(l=0)}(a, T)=-\frac{16\left(k_{B} T\right)^{2}}{\pi^{2} \hbar c\left(\pi \alpha+2 \tilde{v}_{F}\right)} \int_{0}^{\infty} d k_{\perp} \frac{\rho_{\mathrm{TM}}^{2}}{e^{2 k_{\perp} a}-\rho_{\mathrm{TM}}^{2}} \int_{0}^{1} d x \ln \left[1+e^{-2 B_{0} \sqrt{x(1-x)}}\right] \\
& \Delta_{T}^{(2)} \mathcal{F}_{\mathrm{TE}}^{(l=0)}(a, T)=\frac{32 k_{B} T}{\pi^{2}\left(\pi \alpha \tilde{v}_{F}+2\right)} \int_{0}^{\infty} k_{\perp} d k_{\perp} \frac{\rho_{\mathrm{TE}}^{2}}{e^{2 k_{\perp} a}-\rho_{\mathrm{TE}}^{2}} \int_{0}^{1} \frac{\sqrt{x(1-x)} d x}{e^{2 B_{0} \sqrt{x(1-x)}}+1}
\end{aligned}
$$

We consider first the contribution of the TM mode. For this purpose, we introduce the dimensionless integration variable $y=2 a k_{\perp}$ and rewrite the first formula in Eq. (51) in the form

$$
\Delta_{T}^{(2)} \mathcal{F}_{\mathrm{TM}}^{(l=0)}(a, T)=-\frac{8\left(k_{B} T\right)^{2}}{\pi^{2} \hbar c a\left(\pi \alpha+2 \tilde{v}_{F}\right)} Y_{\mathrm{TM}}(a, T)
$$

where

$$
Y_{\mathrm{TM}}(a, T)=\int_{0}^{\infty} d y \frac{\rho_{\mathrm{TM}}^{2}}{e^{y}-\rho_{\mathrm{TM}}^{2}} \int_{0}^{1} d x \ln \left[1+e^{-A y \sqrt{x(1-x)}}\right],
$$

and, according to Eq. (34),

$$
A=\frac{\hbar v_{F}}{2 a k_{B} T}=\frac{T_{\mathrm{eff}}^{(g)}}{T} \gg 1 .
$$

Using the series expansions and calculating the integral with respect to $y$, Eq. (53) is rewritten as

$$
\begin{aligned}
& Y_{\mathrm{TM}}(a, T)=\sum_{n=1}^{\infty} \rho_{\mathrm{TM}}^{2 n} \sum_{k=1}^{\infty} \frac{(-1)^{k+1}}{k} \int_{0}^{1} d x \int_{0}^{\infty} d y e^{-n y-A k y \sqrt{x(1-x)}} \\
& =\sum_{n=1}^{\infty} \rho_{\mathrm{TM}}^{2 n} \sum_{k=1}^{\infty} \frac{(-1)^{k+1}}{k} \int_{0}^{1} \frac{d x}{n+A k \sqrt{x(1-x)}} .
\end{aligned}
$$

Taking into account that $A \rightarrow \infty$ when $T \rightarrow 0$, one can write

$$
Y_{\mathrm{TM}}(a, T)=\frac{1}{A} \sum_{n=1}^{\infty} \rho_{\mathrm{TM}}^{2 n} \sum_{k=1}^{\infty} \frac{(-1)^{k+1}}{k^{2}} \int_{0}^{1} \frac{d x}{\sqrt{x(1-x)}}=\frac{1}{A} \frac{\rho_{\mathrm{TM}}^{2}}{1-\rho_{\mathrm{TM}}^{2}} \frac{\pi^{3}}{12}
$$

Note that an exact calculation of the integral in Eq. (55) leads to the same result under the condition (54).

Substituting Eqs. (37) and (156) in Eq. (52), one arrives at

$$
\Delta_{T}^{(2)} \mathcal{F}_{\mathrm{TM}}^{(l=0)}(a, T)=-\frac{\left(k_{B} T\right)^{3}}{\left(\hbar v_{F}\right)^{2}} \frac{\pi^{3} \alpha^{2}}{3\left(\pi \alpha+2 \tilde{v}_{F}\right)\left(\pi \alpha+\tilde{v}_{F}\right)} .
$$

From the comparison of Eqs. (45) (the first line) and (57), it is seen that the thermal corrections $\Delta_{T}^{(1)} \mathcal{F}_{\mathrm{TM}}$ and $\Delta_{T}^{(2)} \mathcal{F}_{\mathrm{TM}}^{(l=0)}$ have the same sign, corresponding to attraction, and their absolute values are of the same order of magnitude. 
Now we return to the contribution of the TE mode to $\Delta_{T}^{(2)} \mathcal{F}^{(l=0)}$ defined in the second formula of Eq. (51). We rewrite this formula in terms of the variable $y$ using Eq. (377) and neglecting by $\pi \alpha \tilde{v}_{F}$ in comparison with 2 . The result is

$$
\Delta_{T}^{(2)} \mathcal{F}_{\mathrm{TE}}^{(l=0)}(a, T)=\frac{k_{B} T \alpha^{2} \tilde{v}_{F}^{2}}{a^{2}} Y_{\mathrm{TE}}(a, T)
$$

where

$$
Y_{\mathrm{TE}}(a, T)=\int_{0}^{\infty} y d y e^{-y} \int_{0}^{1} d x \frac{\sqrt{x(1-x)}}{e^{A y \sqrt{x(1-x)}}+1} .
$$

By expanding in the power series and integrating with respect to $y$, one finds

$$
\begin{gathered}
Y_{\mathrm{TE}}(a, T)=\sum_{k=1}^{\infty}(-1)^{k+1} \int_{0}^{1} d x \sqrt{x(1-x)} \int_{0}^{\infty} y d y e^{-y-A k y \sqrt{x(1-x)}} \\
=\sum_{k=1}^{\infty}(-1)^{k+1} \int_{0}^{1} d x \frac{\sqrt{x(1-x)}}{[1+A k \sqrt{x(1-x)}]^{2}} .
\end{gathered}
$$

Taking into account Eq. (54), we can neglect by the unity in the denominator and write

$$
Y_{\mathrm{TE}}(a, T)=\frac{1}{A^{2}} \sum_{k=1}^{\infty} \frac{(-1)^{k+1}}{k^{2}} \int_{0}^{1} \frac{d x}{\sqrt{x(1-x)}} .
$$

Integrating with respect to $x$ and summing in $k$, one arrives at

$$
Y_{\mathrm{TE}}(a, T)=\frac{\pi^{3}}{12 A^{2}}=\frac{\pi^{3}}{12}\left(\frac{2 a k_{B} T}{\hbar v_{F}}\right)^{2} .
$$

Substituting this result in Eq. (58), we find

$$
\Delta_{T}^{(2)} \mathcal{F}_{\mathrm{TE}}^{(l=0)}(a, T)=\frac{\left(k_{B} T\right)^{3}}{(\hbar c)^{2}} \frac{\pi^{3} \alpha^{2}}{3}
$$

Thus, the TE contribution to the thermal correction is again of opposite sign to the TM contribution (57). From Eqs. (57) and (63), we have

$$
\frac{\Delta_{T}^{(2)} \mathcal{F}_{\mathrm{TE}}^{(l=0)}}{\left|\Delta_{T}^{(2)} \mathcal{F}_{\mathrm{TM}}^{(l=0)}\right|}=\tilde{v}_{F}^{2}\left(\alpha \pi+2 \tilde{v}_{F}\right)\left(\alpha \pi+\tilde{v}_{F}\right) \ll 1,
$$

i.e., the TE contribution to this part of the thermal correction is again much smaller than the TM contribution. 


\section{B. Contribution of all terms with nonzero Matsubara frequencies}

Now we consider the contribution to the thermal correction $\Delta_{T}^{(2)} \mathcal{F}$ due to all nonzero Matsubara frequencies. We again represent the quantity of our interest as the sum of TM and TE modes

$$
\Delta_{T}^{(2)} \mathcal{F}^{(l \geq 1)}(a, T)=\Delta_{T}^{(2)} \mathcal{F}_{\mathrm{TM}}^{(l \geq 1)}(a, T)+\Delta_{T}^{(2)} \mathcal{F}_{\mathrm{TE}}^{(l \geq 1)}(a, T),
$$

where according to Eq. (16)

$$
\begin{aligned}
& \Delta_{T}^{(2)} \mathcal{F}_{\mathrm{TM}}^{(l \geq 1)}(a, T)=-\frac{2 k_{B} T}{\pi} \sum_{l=1}^{\infty} \int_{0}^{\infty} k_{\perp} d k_{\perp} \\
& \times \frac{\tilde{q}_{l}}{\pi \alpha q_{l}+2 \tilde{q}_{l}} \frac{r_{\mathrm{TM}}^{(0)^{2}}\left(i \xi_{l}, k_{\perp}\right)}{e^{2 q_{l} a}-r_{\mathrm{TM}}^{(0)}\left(i \xi_{l}, k_{\perp}\right)} \frac{\Delta_{T} \Pi_{00}\left(i \xi_{l}, k_{\perp}\right)}{\Pi_{00}^{(0)}\left(i \xi_{l}, k_{\perp}\right)}, \\
& \Delta_{T}^{(2)} \mathcal{F}_{\mathrm{TE}}^{(l \geq 1)}(a, T)=-\frac{2 k_{B} T}{\pi} \sum_{l=1}^{\infty} \int_{0}^{\infty} k_{\perp} d k_{\perp} \\
& \times \frac{q_{l}}{\pi \alpha \tilde{q}_{l}+2 q_{l}} \frac{r_{\mathrm{TE}}^{(0)}{ }^{2}\left(i \xi_{l}, k_{\perp}\right)}{e^{2 q_{l} a}-r_{\mathrm{TE}}^{(0)}\left(i \xi_{l}, k_{\perp}\right)} \frac{\Delta_{T} \Pi\left(i \xi_{l}, k_{\perp}\right)}{\Pi^{(0)}\left(i \xi_{l}, k_{\perp}\right)} .
\end{aligned}
$$

As shown in Appendix A, at low temperature, satisfying Eq. (34), and for $l \geq 1$, Eq. (10) leads to

$$
\begin{aligned}
& \Delta_{T} \Pi_{00}\left(i \xi_{l}, k_{\perp}\right)=\frac{6 \zeta(3) \alpha \hbar k_{\perp}^{2}}{\tilde{q}_{l} B_{l}^{3}}, \\
& \Delta_{T} \Pi\left(i \xi_{l}, k_{\perp}\right)=\frac{12 \zeta(3) \alpha \hbar k_{\perp}^{2} \tilde{q}_{l}}{B_{l}^{3}}\left(\frac{3 \xi_{l}^{2}}{2 c^{2} \tilde{q}_{l}^{2}}-1\right) .
\end{aligned}
$$

We begin with the contribution of the TM mode given by the first formula in Eq. (66)). From Eqs. (67) and (7), using the variable $y$ defined in Eq. (23) and the effective temperature defined in Eq. (24), one obtains

$$
\frac{\Delta_{T} \Pi_{00}}{\Pi_{00}^{(0)}}=\frac{48 \zeta(3)}{\pi \tilde{g}_{l}^{3}}\left(\frac{T}{T_{\mathrm{eff}}}\right)^{3}
$$

where $\tilde{g}_{l}$ is defined in Eq. (28).

Now we rewrite the first formula in Eq. (66) in terms of the variable $y$ and use Eq. (68). The result is

$$
\Delta_{T}^{(2)} \mathcal{F}_{\mathrm{TM}}^{(l \geq 1)}(a, T)=-\frac{24 \zeta(3) k_{B} T}{\pi^{2} a^{2}}\left(\frac{T}{T_{\mathrm{eff}}}\right)^{3} X_{\mathrm{TM}}(a, T),
$$


where

$$
X_{\mathrm{TM}}(a, T)=\sum_{l=1}^{\infty} \int_{\tau l}^{\infty} \frac{y d y}{\tilde{g}_{l}^{2}} \frac{1}{\pi \alpha y+2 \tilde{g}_{l}} \frac{r_{\mathrm{TM}}^{(0)^{2}}}{e^{y}-r_{\mathrm{TM}}^{(0)}}
$$

and $r_{\mathrm{TM}}^{(0)} \equiv r_{\mathrm{TM}}^{(0)}\left(y, \zeta_{l}\right)$ is defined in Eq. (27).

Expanding under the integral in powers of ${r_{\mathrm{TM}}^{(0)}}^{2} e^{-y}$, we can rewrite Eq. (70) as

$$
X_{\mathrm{TM}}(a, T)=\sum_{l=1}^{\infty} \sum_{n=1}^{\infty} \int_{\tau l}^{\infty} \frac{y d y}{\tilde{g}_{l}^{2}} \frac{r_{\mathrm{TM}}^{(0)}{ }^{2 n} e^{-n y}}{\pi \alpha y+2 \tilde{g}_{l}} .
$$

It is convenient to use Eq. (27) for $r_{\mathrm{TM}}^{(0)}$ and introduce a new integration variable $x=$ $y /(\tau l)$. Then, Eq. (71) takes the form

$$
X_{\mathrm{TM}}(a, T)=\sum_{l=1}^{\infty} \sum_{n=1}^{\infty} \frac{e^{-n l \tau}}{\tau l} Q_{n}(\tau l),
$$

where

$$
Q_{n}(\tau l)=\int_{1}^{\infty} \frac{x d x}{\left(\tilde{v}_{F}^{2} x^{2}+1\right)}\left(\frac{\alpha \pi x}{\alpha \pi x+2 \sqrt{\tilde{v}_{F}^{2} x^{2}+1}}\right)^{2 n} \frac{e^{-n l \tau(x-1)}}{\alpha \pi x+2 \sqrt{\tilde{v}_{F}^{2} x^{2}+1}} .
$$

We are interested in finding the leading term of the quantity $Q_{n}(\tau l)$ when $\tau$ goes to zero. This is given by

$$
Q_{n}(0)=\int_{1}^{\infty} \frac{x d x}{\left(\tilde{v}_{F}^{2} x^{2}+1\right)}\left(\frac{\alpha \pi x}{\alpha \pi x+2 \sqrt{\tilde{v}_{F}^{2} x^{2}+1}}\right)^{2 n} \frac{1}{\alpha \pi x+2 \sqrt{\tilde{v}_{F}^{2} x^{2}+1}} .
$$

The correction terms to Eq. (74) due to nonzero $\tau$ go to zero when $\tau$ vanishes. This is seen from the following:

$$
\left|Q_{n}(\tau l)-Q_{n}(0)\right|<\frac{1}{\tilde{v}_{F}^{2}}\left(\frac{\alpha \pi}{\alpha \pi+2 \tilde{v}_{F}}\right)^{2 n} \frac{1}{\alpha \pi+2 \tilde{v}_{F}} \int_{1}^{\infty} \frac{d x}{x^{2}}\left[1-e^{-n l \tau(x-1)}\right]
$$

and from the fact that the integral entering the right-hand side of this equation goes to zero when $\tau \rightarrow 0$ :

$$
-n l \tau e^{n l \tau} \operatorname{Ei}(-n l \tau) \rightarrow 0 .
$$

We find the leading term of the quantity (72) in the limiting case $\tau \rightarrow 0$ by substituting $Q_{n}(0)$ in place of $Q_{n}(\tau l)$. In so doing, the summation in $l$ is performed according to

$$
\sum_{l=1}^{\infty} \frac{e^{-n l \tau}}{l}=-\ln \left(1-e^{-n \tau}\right) \approx-\ln (\tau n)=-\ln \tau-\ln n,
$$


where for obtaining the leading term one should keep only the first contribution on the right-hand side. Performing also the trivial summation in $n$, one obtains

$$
X_{\mathrm{TM}}(a, T)=-\frac{\ln \tau}{\tau} C_{\mathrm{TM}}
$$

where the constant $C_{\mathrm{TM}}$ is given by

$$
C_{\mathrm{TM}}=\frac{\alpha^{2} \pi^{2}}{4} \int_{1}^{\infty} \frac{x^{3} d x}{\left(\tilde{v}_{F}^{2} x^{2}+1\right)^{3 / 2}} \frac{1}{\left(\alpha \pi x+\sqrt{\tilde{v}_{F}^{2} x^{2}+1}\right)\left(\alpha \pi x+2 \sqrt{\tilde{v}_{F}^{2} x^{2}+1}\right)} .
$$

The numerical integration in Eq. (79) results in $C_{\mathrm{TM}} \approx 1.3 \times 10^{4}$.

Substituting Eq. (78) in Eq. (69), we arrive at

$$
\Delta_{T}^{(2)} \mathcal{F}_{\mathrm{TM}}^{(l \geq 1)}(a, T)=\frac{\left(k_{B} T\right)^{3}}{(\hbar c)^{2}} \ln \left(\frac{a k_{B} T}{\hbar c}\right) \frac{48 \zeta(3)}{\pi^{3}} C_{\mathrm{TM}} .
$$

Note that we have omitted the factor $4 \pi$ under the logarithm because it contributes to the next after the leading term of order of $T^{3}$.

Now we continue with the contribution of the TE mode to the thermal correction $\Delta_{T}^{(2)} \mathcal{F}^{(l \geq 1)}$. From the second formulas of Eqs. (67) and (77), one obtains

$$
\frac{\Delta_{T} \Pi}{\Pi^{(0)}}=\frac{96 \zeta(3)}{\pi \tilde{g}_{l}^{3}}\left(\frac{T}{T_{\text {eff }}}\right)^{3}\left(\frac{3 \tau^{2} l^{2}}{2 \tilde{g}_{l}^{2}}-1\right) .
$$

Substituting this results in the second formula of Eq. (66), we find

$$
\Delta_{T}^{(2)} \mathcal{F}_{\mathrm{TE}}^{(l \geq 1)}(a, T)=-\frac{48 \zeta(3) k_{B} T}{\pi^{2} a^{2}}\left(\frac{T}{T_{\text {eff }}}\right)^{3} X_{\mathrm{TE}}(a, T),
$$

where

$$
X_{\mathrm{TE}}(a, T)=\sum_{l=1}^{\infty} \int_{\tau l}^{\infty} \frac{y^{2} d y}{\tilde{g}_{l}^{3}} \frac{1}{\pi \alpha \tilde{g}_{l}+2 y} \frac{r_{\mathrm{TE}}^{(0)^{2}}}{e^{y}-r_{\mathrm{TE}}^{(0)^{2}}}\left(\frac{3 \tau^{2} l^{2}}{2 \tilde{g}_{l}^{2}}-1\right)
$$

and $r_{\mathrm{TE}}^{(0)} \equiv r_{\mathrm{TE}}^{(0)}\left(y, \zeta_{l}\right)$ is defined in Eq. (27)).

Similarly to the case of TM mode, we expand in powers of $r_{\mathrm{TE}}^{(0)} e^{-y}$ and obtain

$$
X_{\mathrm{TE}}(a, T)=\sum_{l=1}^{\infty} \sum_{n=1}^{\infty} \int_{\tau l}^{\infty} \frac{y^{2} d y}{\tilde{g}_{l}^{3}} \frac{r_{\mathrm{TE}}^{(0)^{2 n}} e^{-n y}}{\pi \alpha \tilde{g}_{l}+2 y}\left(\frac{3 \tau^{2} l^{2}}{2 \tilde{g}_{l}^{2}}-1\right)
$$

Introducing the integration variable $x=y /(\tau l)$ and using Eq. (27) for $r_{\mathrm{TE}}^{(0)}$, one arrives at

$$
X_{\mathrm{TE}}(a, T)=\sum_{l=1}^{\infty} \sum_{n=1}^{\infty} \frac{e^{-n l \tau}}{\tau l} G_{n}(\tau l),
$$


where

$$
\begin{aligned}
& G_{n}(\tau l)=\int_{1}^{\infty} \frac{x^{2} d x}{\left(\tilde{v}_{F}^{2} x^{2}+1\right)^{3 / 2}}\left(\frac{\alpha \pi \sqrt{\tilde{v}_{F}^{2} x^{2}+1}}{\alpha \pi \sqrt{\tilde{v}_{F}^{2} x^{2}+1}+2 x}\right)^{2 n} \\
& \times \frac{e^{-n l \tau(x-1)}}{\alpha \pi \sqrt{\tilde{v}_{F}^{2} x^{2}+1}+2 x}\left[\frac{3}{2\left(\tilde{v}_{F}^{2} x^{2}+1\right)}-1\right] .
\end{aligned}
$$

Similarly to the case of the TM mode, the leading term of Eq. (86) at small $\tau$ is given by the value of $G_{n}$ at $\tau=0$. Substituting $G_{n}(0)$ in place of $G_{n}(\tau l)$ in Eq. (85) and performing the summations in $l$ and $n$, we obtain

$$
X_{\mathrm{TE}}(a, T)=-\frac{\ln \tau}{\tau} C_{\mathrm{TE}}
$$

where

$$
\begin{aligned}
C_{\mathrm{TE}} & =\frac{\alpha^{2} \pi^{2}}{4} \int_{1}^{\infty} \frac{d x}{\sqrt{\tilde{v}_{F}^{2} x^{2}+1}\left(\alpha \pi \sqrt{\tilde{v}_{F}^{2} x^{2}+1}+x\right)} \\
\times & \frac{1}{\alpha \pi \sqrt{\tilde{v}_{F}^{2} x^{2}+1}+2 x}\left[\frac{3}{2\left(\tilde{v}_{F}^{2} x^{2}+1\right)}-1\right] .
\end{aligned}
$$

According to the results of the numerical integration, $C_{\mathrm{TE}} \approx 1.1 \times 10^{-4}$.

Finally, the substitution of Eq. (87) in Eq. (82) leads to

$$
\Delta_{T}^{(2)} \mathcal{F}_{\mathrm{TE}}^{(l \geq 1)}(a, T)=\frac{\left(k_{B} T\right)^{3}}{(\hbar c)^{2}} \ln \left(\frac{a k_{B} T}{\hbar c}\right) \frac{96 \zeta(3)}{\pi^{3}} C_{\mathrm{TE}}
$$

It is seen that

$$
\frac{\Delta_{T}^{(2)} \mathcal{F}_{\mathrm{TE}}^{(l \geq 1)}(a, T)}{\Delta_{T}^{(2)} \mathcal{F}_{\mathrm{TM}}^{(l \geq 1)}(a, T)}=2 \frac{C_{\mathrm{TE}}}{C_{\mathrm{TM}}} \approx 1.7 \times 10^{-8},
$$

i.e., the contribution of the TE mode is again negligibly small as compared to the contribution of the TM mode.

\section{LOW-TEMPERATURE BEHAVIOR OF THE CASIMIR FREE ENERGY, ENTROPY AND PRESSURE FOR TWO GRAPHENE SHEETS}

According to the above results, at low temperatures, satisfying the condition (34), the leading term of the thermal correction to the Casimir energy is given by Eq. (80). This term is determined by an explicit dependence of the polarization tensor on temperature as a parameter and originates from all contributions to the Lifshitz formula with nonzero Matsubara frequencies. We have shown also that at low temperature the magnitudes of 
all other parts of the thermal correction to the Casimir energy (determined by the zerotemperature polarization tensor calculated at the Matsubara frequencies and by the thermal correction to it calculated at zero frequency) are of the next-to-leading order. In all cases the TM mode gives the major contribution to the result.

Thus, in accordance with Eq. (21), the Casimir free energy at low temperature can be written as

$$
\mathcal{F}(a, T)=E(a)+\frac{\left(k_{B} T\right)^{3}}{(\hbar c)^{2}} \ln \left(\frac{a k_{B} T}{\hbar c}\right) \frac{48 \zeta(3)}{\pi^{3}} C_{\mathrm{TM}}
$$

where the Casimir energy $E(a)$ at $T=0$ is defined in Eq. (17) and the coefficient $C_{\mathrm{TM}}$ is given in Eq. (79) . As is seen from Eq. (91), the thermal correction has the same (negative) sign as the Casimir energy, which corresponds to an attraction.

Now we are in a position to calculate the Casimir entropy of two graphene sheets in the limit of low temperatures. This is of interest in connection with the problems arising for two Casimir plates made of metals or dielectrics (see Sec. I). From Eq. (91) we obtain the leading contribution to the entropy in the form

$$
S(a, T)=-\frac{\partial \mathcal{F}(a, T)}{\partial T}=-\left(\frac{k_{B} T}{\hbar c}\right)^{2} \ln \left(\frac{a k_{B} T}{\hbar c}\right) \frac{144 \zeta(3) k_{B}}{\pi^{3}} C_{\mathrm{TM}} .
$$

As is seen in Eq. (92), the Casimir entropy of two graphene sheets is positive. With decreasing $T$ we have

$$
\lim _{T \rightarrow 0} S(a, T)=0,
$$

which means that the Nernst heat theorem is satisfied. Thus, the Lifshitz theory of the Casimir interaction between two graphene sheets is in agreement with thermodynamics in spite of the fact that some of its properties (specifically, large thermal effect at short separations and almost zero contribution of the TE mode) are reminiscent of that of metals described by the Drude model. It is not surprising, however, that for graphene the theory turns out to be thermodynamically consistent. The reason is that the reflection coefficients (3) are expressed via the polarization tensor (6), or equivalent nonlocal dielectric permittivities (5), which are calculated starting from the first principles of quantum electrodynamics at nonzero temperature.

As opposed to next-to-leading-order contributions to the thermal correction presented in Eqs. (45), (57), and (63), which are independent on the separation between graphene sheets, the leading term (89) depends on $a$. This makes possible to obtain an asymptotic 
expression for the Casimir pressure between two graphene sheets at vanishing temperature. From Eq. (91) one obtains

$$
P(a, T)=-\frac{\partial \mathcal{F}(a, T)}{\partial a}=P_{0}(a)-\frac{\left(k_{B} T\right)^{3}}{(\hbar c)^{2}} \frac{48 \zeta(3)}{\pi^{3}} \frac{C_{\mathrm{TM}}}{a} .
$$

As is seen from this equation, at low temperature the thermal correction to the Casimir pressure is inversely proportional to the separation distance.

At the end of this section, we characterize the experimental situation. By now there is only one experiment on measuring the Casimir interaction between a Au-coated sphere and a graphene-coated substrate [70]. Using the Lifshitz theory with reflection coefficients expressed via the polarization tensor of graphene and dielectric permittivity of substrate, the measurement data were found to be in very good agreement with the calculation results [11]. Thus, as it was already demonstrated for metals and dielectrics, the measurement results for graphene again confirm the thermodynamically consistent theory.

\section{CONCLUSIONS AND DISCUSSION}

In the foregoing, we have investigated the low-temperature behavior of the Casimir free energy between two graphene sheets. This was done in the framework of a fundamental theory using the polarization tensor in $(2+1)$-dimensional space-time defined over the entire plane of complex frequency. The thermal correction to the Casimir energy was separated in two parts: the first one determined by the polarization tensor at zero temperature calculated at the discrete Matsubara frequencies, and the second one determined by the thermal correction to the polarization tensor. The second part of the thermal correction was subdivided into the contributions of the zero-frequency term of the Lifshitz formula and all terms with nonzero Matsubara frequencies.

Using the analytic asymptotic expansions in powers of small parameters, we have shown that at all temperatures, which are much smaller than the effective temperature of graphene defined in Eq. (34), the leading terms in the first part of the thermal correction to the Casimir energy and in the zero-frequency contribution to the second part behave as $\sim$ $T^{3}$. The leading term in the second part of the thermal correction, originating from all nonzero Matsubara frequencies, behaves as $\sim T^{3} \ln T$. Thus, it determines the temperature dependence of the Casimir free energy at low temperature. 
Note that at higher temperatures the situation is quite different. Thus, at $T=300 \mathrm{~K}$ the dominant contributions to the thermal effect are given by the first part of the thermal correction and by the zero-frequency term in the second part of the thermal correction [33]. These are the contributions which, according to our results, are of the next-to-leading order at low temperatures. In so doing, at both low and room temperatures the contributions of the TE mode to all the results are much smaller in magnitude than the respective contributions of the TM mode and can be neglected.

The obtained analytic expression for the Casimir free energy of two graphene sheets at low temperature was used to calculate the low-temperature behavior of the Casimir entropy and pressure. It was shown that the Casimir entropy behaves as $\sim T^{2} \ln T$ and, thus, goes to zero when the temperature vanishes in accordance with the Nernst heat theorem. The thermal correction to the Casimir pressure between two graphene sheets behaves at low temperature as $\sim T^{3} / a$, i.e., slowly increases with decreasing $a$. This should be compared with the thermal correction to the Casimir pressure between Drude metals, which behaves at low $T$ as $P_{D} \sim T / a^{3}[1,38]$.

The obtained results shed new light on the problem of theoretical description of free charge carriers in the Casimir physics. This problem at the moment is not of only academic character. The point is that in the difference force measurements [53 57], as well as for the Casimir free energy and pressure of thin metallic films [80 82], the predictions of the Drude and plasma model approaches are recently shown to differ by up to a factor of several thousands. As we have shown here, an experimentally consistent theory for graphene, using the polarization tensor in $(2+1)$-dimensional space-time, is in agreement with the requirements of thermodynamics similar to experimentally consistent approaches for metallic and dielectric test bodies. This confirms that the thermodynamic test is important for future resolution of the problem of relaxation properties of free charge carriers in the Casimir physics.

\section{Acknowledgments}

The authors of this work acknowledge $\mathrm{CNPq}$ (Brazil) for partial financial support (the Grants 307596/2015-0 and 308150/2015-5). They are also indebted to M. Bordag for helpful discussions, for reading the manuscript, and for useful corrections and suggestions. The work 
of V.M.M. was partially supported by the Russian Government Program of Competitive Growth of Kazan Federal University. G.L.K. and V.M.M. are grateful to the Department of Physics of the Federal University of Paraíba (João Pessoa, Brazil) for kind hospitality.

\section{Appendix A}

Here, we derive the asymptotic expressions for the thermal corrections, $\Delta_{T} \Pi_{00}$ and $\Delta_{T} \Pi$, valid under the conditions (33) and (34) at all nonzero Matsubara frequencies. We start from $\Delta_{T} \Pi_{00}\left(i \xi_{l} . k_{\perp}\right)$ presented in the first formula of Eq. (10) and introduce the following notation for the integral entering this formula

$$
I_{00}=\int_{0}^{\infty} \frac{d u}{e^{B_{l} u}+1}\left[1-\frac{1}{\sqrt{2}} \sqrt{Z_{00}(u)}\right],
$$

where

$$
Z_{00}=\sqrt{\left(1+u^{2}\right)^{2}-4 \frac{\tilde{v}_{F}^{2} k_{\perp}^{2} u^{2}}{\tilde{q}_{l}^{2}}}+1-u^{2} .
$$

The major contribution to the integral (A1) is given by $u$ satisfying the condition $B_{l} u \sim 1$. Taking into account that due to Eq. (34)

$$
B_{l} \equiv \frac{\hbar c \tilde{q}_{l}}{2 k_{B} T}>\frac{\hbar c \tilde{v}_{F} k_{\perp}}{2 k_{B} T} \sim \frac{\hbar v_{F}}{4 a k_{B} T} \gg 1
$$

we conclude that the major contribution to the integral (A1) is given by $u \ll 1$.

When it is considered that $\tilde{v}_{F}^{2} k_{\perp}^{2} / \tilde{q}_{l}^{2}<1$, in the region of $u$, giving the major contribution to the integral (A1), the following condition is satisfied:

$$
\frac{\tilde{v}_{F}^{2} k_{\perp}^{2}}{\tilde{q}_{l}^{2}} u^{2} \ll 1 .
$$

Now we expand the quantity $Z_{00}(u)$ in powers of the small parameter (A4) and obtain

$$
Z_{00}(u)=1+u^{2}-2 \frac{\tilde{v}_{F}^{2} k_{\perp}^{2}}{\tilde{q}_{l}^{2}} \frac{u^{2}}{1+u^{2}}+1-u^{2} .
$$

Then, neglecting $u^{2}$ as compared to unity in the denominator, we find

$$
Z_{00}(u)=2\left(1-\frac{\tilde{v}_{F}^{2} k_{\perp}^{2} u^{2}}{\tilde{q}_{l}^{2}}\right) .
$$

Substituting Eq. (A6) in Eq. (A1), expanding the square root in the same small parameter (A4), and integrating with respect to $u$, one arrives at

$$
I_{00}=\frac{\tilde{v}_{F}^{2} k_{\perp}^{2}}{2 \tilde{q}_{l}^{2}} \int_{0}^{\infty} \frac{u^{2} d u}{e^{B_{l} u}+1}=\frac{3 \zeta(3) \tilde{v}_{F}^{2} k_{\perp}^{2}}{4 \tilde{q}_{l}^{2} B_{l}^{3}} .
$$


Finally, we substitute this equation in the first formula of Eq. (10) and arrive at the first formula of Eq. (67).

We emphasize that the above derivation is valid only for nonzero Matsubara frequencies with $l \geq 1$. The point is that for $l=0$ one has $\tilde{v}_{F} k_{\perp}=\tilde{q}_{0}$ and, as a result, we have the term $\left(1-u^{2}\right)^{2}$ under the square root in Eq. (A2). In this case, Eq. (A5) would be applicable only for $u<1$. This makes impossible the substitution of Eq. (A6) in Eq. (A1).

Note that if the higher-order terms in the small parameter (A4) are taken into account in Eq. (A5), which leads to integrals of the form

$$
I_{00}^{(k)}=\int_{0}^{\infty} \frac{u^{2 k} d u}{e^{B_{l} u}+1}=\frac{C_{k}}{B_{l}^{2 k+1}}
$$

where $k \geq 2$. This would result in the higher-order corrections $\sim\left(T / T_{\text {eff }}\right)^{2 k+1}$ to $\Delta_{T} \Pi_{00}$, which are omitted in our calculation.

Now we consider the thermal correction $\Delta_{T} \Pi\left(i \xi_{l}, k_{\perp}\right)$ presented in the second formula of Eq. (10). The integral entering this formula can be rewritten in the form

$$
I=\int_{0}^{\infty} \frac{d u}{e^{B_{l} u}+1}\left[-\frac{\xi_{l}^{2}}{c^{2}}+\frac{\tilde{q}_{l}^{2}}{\sqrt{2}} \sqrt{Z_{00}(u)} Z(u)\right]
$$

where $Z_{00}$ is defined in Eq. (A6) and

$$
Z(u)=1-\frac{\tilde{v}_{F}^{2} k_{\perp}^{2}}{\tilde{q}_{l}^{2} \sqrt{\left(1+u^{2}\right)^{2}-4 \frac{\tilde{v}_{F}^{2} k_{\perp}^{2} u^{2}}{\tilde{q}_{l}^{2}}}} .
$$

We again use the small parameter (A4) and the expansion (A6) of $Z_{00}$ in powers of this parameter. Expanding also the quantity $Z$ in powers of the same parameter, we obtain

$$
Z(u)=1-\frac{\tilde{v}_{F}^{2} k_{\perp}^{2}}{\tilde{q}_{l}^{2}\left(1+u^{2}\right)}\left[1+2 \frac{\tilde{v}_{F}^{2} k_{\perp}^{2} u^{2}}{\tilde{q}_{l}^{2}\left(1+u^{2}\right)^{2}}\right] .
$$

Furthermore, expansion of Eq. (A11) up to the second order in the small parameter $u$ results in

$$
Z(u)=\frac{\xi_{l}^{2}}{c^{2} \tilde{q}_{l}^{2}}+\frac{\tilde{v}_{F}^{2} k_{\perp}^{2} u^{2}}{\tilde{q}_{l}^{2}}\left(1-2 \frac{\tilde{v}_{F}^{2} k_{\perp}^{2}}{\tilde{q}_{l}^{2}}\right) .
$$

Substituting Eqs. (A6) and (A12) in Eq. (A9), one arrives at

$$
I=-\tilde{v}_{F}^{2} k_{\perp}^{2}\left(1-\frac{3 \xi_{l}^{2}}{2 c^{2} \tilde{q}_{l}^{2}}\right) \int_{0}^{\infty} \frac{u^{2} d u}{e^{B_{l} u}+1}=-\frac{3 \zeta(3) \tilde{v}_{F}^{2} k_{\perp}^{2}}{2 B_{l}^{3}}\left(1-\frac{3 \xi_{l}^{2}}{2 c^{2} \tilde{q}_{l}^{2}}\right) .
$$

Then, from the second formula of Eq. (10), we arrive at the second formula of Eq. (67). 
Note that the expansion terms of $Z_{00}(u)$ and $Z(u)$, which are of higher orders in $u$ than in Eqs. (A6) and (A12), lead to correction terms of the same form, as in Eq. (A8), and to higher-order corrections to $\Delta_{T} \Pi$ than the leading term presented in Eq. (67).

[1] M. Bordag, G. L. Klimchitskaya, U. Mohideen, and V. M. Mostepanenko, Advances in the Casimir Effect (Oxford University Press, Oxford, 2015).

[2] E. M. Lifshitz and L. P. Pitaevskii, Statistical Physics, Part II (Pergamon, Oxford, 1980).

[3] J. F. Babb, G. L. Klimchitskaya, and V. M. Mostepanenko, Phys. Rev. A 70, 042901 (2004).

[4] M. Antezza, L. P. Pitaevskii, and S. Stringari, Phys. Rev. A 70, 053619 (2004).

[5] A. O. Caride, G. L. Klimchitskaya, V. M. Mostepanenko, and S. I. Zanette, Phys. Rev. A 71, 042901 (2005).

[6] S. Y. Buhmann and S. Scheel, Phys. Rev. Lett. 100, 253201 (2008).

[7] H. Safari, D.-G. Welsch, S. Y. Buhmann, and S. Scheel. Phys. Rev. A 78, 062901 (2008).

[8] V. B. Bezerra, G. L. Klimchitskaya, V. M. Mostepanenko, and C. Romero, Phys. Rev. A 78, 042901 (2008).

[9] F. Chen, G. L. Klimchitskaya, U. Mohideen, and V. M. Mostepanenko, Phys. Rev. Lett. 90, $160404(2003)$.

[10] F. Chen, G. L. Klimchitskaya, V. M. Mostepanenko, and U. Mohideen, Phys. Rev. Lett. 97, $170402(2006)$.

[11] P. J. van Zwol, G. Palasantzas, and J. Th. M. De Hosson, Phys. Rev. B 77, 075412 (2008).

[12] S. de Man, K. Heeck, and D. Iannuzzi, Phys. Rev. A 82, 062512 (2010).

[13] G. Torricelli, P. J. van Zwol, O. Shpak, C. Binns, G. Palasantzas, B. J. Kooi, V. B. Svetovoy, and M. Wittig, Phys. Rev. A 82, 010101(R) (2010).

[14] H. B. Chan, V. A. Aksyuk, R. N. Kleiman, D. J. Bishop, and F. Capasso, Science 291, 1941 (2001).

[15] E. Buks and M. L. Roukes, Phys. Rev. B 63, 033402 (2001).

[16] R. H. French, V. A. Parsegian, R. Podgornik, et al., Rev. Mod. Phys. 82, 1887 (2010).

[17] R. Esquivel-Sirvent and R. Pérez-Pascual, Eur. Phys. J. B 86, 467 (2013).

[18] J. Zou, Z. Marcet, A. W. Rodriguez, M. T. H. Reid, A. P. McCauley, I. I. Kravchenko, T. Lu, Y. Bao, S. G. Johnson, and H. B. Chan, Nature Commun. 4, 1845 (2013). 
[19] M. Bordag, I. V. Fialkovsky, D. M. Gitman, and D. V. Vassilevich, Phys. Rev. B 80, 245406 (2009).

[20] G. Gómez-Santos, Phys. Rev. B 80, 245424 (2009).

[21] D. Drosdoff and L. M. Woods, Phys. Rev. B 82, 155459 (2010).

[22] D. Drosdoff and L. M. Woods, Phys. Rev. A 84, 062501 (2011).

[23] Bo E. Sernelius, Europhys. Lett. 95, 57003 (2011).

[24] I. V. Fialkovsky, V. N. Marachevsky, and D. V. Vassilevich, Phys. Rev. B 84, 035446 (2011).

[25] Bo E. Sernelius, Phys. Rev. B 85, 195427 (2012).

[26] A. D. Phan, L. M. Woods, D. Drosdoff, I. V. Bondarev, and N. A. Viet, Appl. Phys. Lett. 101, 113118 (2012).

[27] M. Bordag, G. L. Klimchitskaya, and V. M. Mostepanenko, Phys. Rev. B 86, 165429 (2012).

[28] M. Chaichian, G. L. Klimchitskaya, V. M. Mostepanenko, and A. Tureanu, Phys. Rev. A 86, $012515(2012)$.

[29] G. L. Klimchitskaya and V. M. Mostepanenko, Phys. Rev. B 87, 075439 (2013).

[30] G. L. Klimchitskaya, V. M. Mostepanenko, and Bo E. Sernelius, Phys. Rev. B 89, 125407 (2014).

[31] G. L. Klimchitskaya and V. M. Mostepanenko, Phys. Rev A 89, 052512 (2014).

[32] G. L. Klimchitskaya and V. M. Mostepanenko, Phys. Rev. B 91, 045412 (2015).

[33] G. L. Klimchitskaya and V. M. Mostepanenko, Phys. Rev B 91, 174501 (2015).

[34] A. H. Castro Neto, F. Guinea, N. M. R. Peres, K. S. Novoselov, and A. K. Geim, Rev. Mod. Phys. 81, 109 (2009).

[35] G. L. Klimchitskaya, U. Mohideen, and V. M. Mostepanenko, Rev. Mod. Phys. 81, 1827 (2009).

[36] V. B. Bezerra, G. L. Klimchitskaya, and V. M. Mostepanenko, Phys. Rev. A 65, 052113 (2002).

[37] V. B. Bezerra, G. L. Klimchitskaya, and V. M. Mostepanenko, Phys. Rev. A 66, 062112 (2002).

[38] V. B. Bezerra, G. L. Klimchitskaya, V. M. Mostepanenko, and C. Romero, Phys. Rev. A 69, $022119(2004)$.

[39] M. Bordag and I. Pirozhenko, Phys. Rev. D 82, 125016 (2010).

[40] G. L. Klimchitskaya and C. C. Korikov, Phys. Rev. A 91, 032119 (2015); 92, 029902(E) 
(2015).

[41] M. Boström and Bo E. Sernelius, Physica A 339, 53 (2004).

[42] J. S. Høye, I. Brevik, S. A. Ellingsen, and J. B. Aarseth, Phys. Rev. E 75, 051127 (2007).

[43] G. L. Klimchitskaya and V. M. Mostepanenko, Phys. Rev. E 77, 023101 (2008).

[44] V. B. Svetovoy and R. Esquivel, Phys. Rev. E 72, 036113 (2005).

[45] B. Geyer, G. L. Klimchitskaya, and V. M. Mostepanenko, Phys. Rev. A 70, 016102 (2004).

[46] R. S. Decca, E. Fischbach, G. L. Klimchitskaya, D. E. Krause, D. López, and V. M. Mostepanenko, Phys. Rev. D 68, 116003 (2003).

[47] R. S. Decca, D. López, E. Fischbach, G. L. Klimchitskaya, D. E. Krause, and V. M. Mostepanenko, Ann. Phys. (N.Y.) 318, 37 (2005).

[48] R. S. Decca, D. López, E. Fischbach, G. L. Klimchitskaya, D. E. Krause, and V. M. Mostepanenko, Phys. Rev. D 75, 077101 (2007).

[49] R. S. Decca, D. López, E. Fischbach, G. L. Klimchitskaya, D. E. Krause, and V. M. Mostepanenko, Eur. Phys. J. C 51, 963 (2007).

[50] C.-C. Chang, A. A. Banishev, R. Castillo-Garza, G. L. Klimchitskaya, V. M. Mostepanenko, and U. Mohideen, Phys. Rev. B 85, 165443 (2012).

[51] A. A. Banishev, G. L. Klimchitskaya, V. M. Mostepanenko, and U. Mohideen, Phys. Rev. Lett. 110, 137401 (2013).

[52] A. A. Banishev, G. L. Klimchitskaya, V. M. Mostepanenko, and U. Mohideen, Phys. Rev. B 88, 155410 (2013).

[53] G. Bimonte, Phys. Rev. Lett. 112, 240401 (2014).

[54] G. Bimonte, Phys. Rev. Lett. 113, 240405 (2014).

[55] G. Bimonte, Phys. Rev. B 91, 205443 (2015).

[56] R. S. Decca, Int. J. Mod. Phys. A 31, 1641024 (2016).

[57] G. Bimonte, D. López, and R. S. Decca, Phys. Rev. B 93, 184434 (2016).

[58] B. Geyer, G. L. Klimchitskaya, and V. M. Mostepanenko, Phys. Rev. D 72, 085009 (2005).

[59] G. L. Klimchitskaya, B. Geyer, and V. M. Mostepanenko, J. Phys. A: Math. Gen. 39, 6495 (2006).

[60] G. L. Klimchitskaya, U. Mohideen, and V. M. Mostepanenko, J. Phys. A: Math. Theor. 41, 432001 (2008).

[61] B. Geyer, G. L. Klimchitskaya, and V. M. Mostepanenko, Ann. Phys. (N.Y.) 323, 291 (2008). 
[62] G. L. Klimchitskaya and C. C. Korikov, J. Phys.: Condens. Matter 27, 214007 (2015).

[63] J. M. Obrecht, R. J. Wild, M. Antezza, L. P. Pitaevskii, S. Stringari, and E. A. Cornell, Phys. Rev. Lett. 98, 063201 (2007).

[64] F. Chen, G. L. Klimchitskaya, V. M. Mostepanenko, and U. Mohideen, Phys. Rev. B 76, $035338(2007)$.

[65] C.-C. Chang, A. A. Banishev, G. L. Klimchitskaya, V. M. Mostepanenko, and U. Mohideen, Phys. Rev. Lett. 107, 090403 (2011).

[66] A. A. Banishev, C.-C. Chang, R. Castillo-Garza, G. L. Klimchitskaya, V. M. Mostepanenko, and U. Mohideen, Phys. Rev. B 85, 045436 (2012).

[67] G. L. Klimchitskaya and V. M. Mostepanenko, J. Phys. A: Math. Theor. 41, 312002 (2008).

[68] B. Arora, H. Kaur, and B. K. Sahoo, J. Phys. B 47, 155002 (2014).

[69] K. Kaur, J. Kaur, B. Arora, and B. K. Sahoo, Phys. Rev. B 90, 245405 (2014).

[70] A. A. Banishev, H. Wen, J. Xu, R. K. Kawakami, G. L. Klimchitskaya, V. M. Mostepanenko, and U. Mohideen, Phys. Rev. B 87, 205433 (2013).

[71] G. L. Klimchitskaya, U. Mohideen, and V. M. Mostepanenko, Phys. Rev B 89, 115419 (2014).

[72] M. Bordag, G. L. Klimchitskaya, V. M. Mostepanenko, and V. M. Petrov, Phys. Rev. D 91, 045037 (2015); 93, 089907(E) (2016).

[73] G. L. Klimchitskaya and V. M. Mostepanenko, Phys. Rev. A 93, 052106 (2016).

[74] G. L. Klimchitskaya, C. C. Korikov, and V. M. Petrov, Phys. Rev. B 92, 125419 (2015); 93, 159906(E) (2016).

[75] G. L. Klimchitskaya and V. M. Mostepanenko, Phys. Rev. B 93, 245419 (2016).

[76] Bo E. Sernelius, J. Phys.: Condens. Matter 27, 214017 (2015).

[77] M. Bordag, I. Fialkovsky, and D. Vassilevich, Phys. Rev. B 93, 075414 (2016).

[78] I. S. Gradshtein and I. M. Ryzhik, Table of Integrals, Series and Products (Academic Press, New York, 1980).

[79] M. Bordag, Adv. Math. Phys., 981586 (2014).

[80] G. L. Klimchitskaya and V. M. Mostepanenko, Phys. Rev. A 92, 042109 (2015).

[81] G. L. Klimchitskaya and V. M. Mostepanenko, Phys. Rev. A 93, 042508 (2016).

[82] G. L. Klimchitskaya and V. M. Mostepanenko, Phys. Rev. B 94, 045404 (2016). 